\title{
Circulating tumor DNA dynamics in advanced breast cancer treated with CDK4/6 inhibition and endocrine therapy
}

Olga Martínez-Sáez ${ }^{1,2,3}$, Tomás Pascual (iD ${ }^{1,2,3,4}$, Fara Brasó-Maristany ${ }^{1,2,3}$, Nuria Chic ${ }^{1,2,3}$, Blanca González-Farré ${ }^{1,3,5}$, Esther Sanfeliu ${ }^{1,3,5}$, Adela Rodríguez ${ }^{2}$, Débora Martínez ${ }^{1,2,3}$, Patricia Galván ${ }^{1,2,3}$, Anna Belén Rodríguez ${ }^{1,2,3}$, Francesco Schettini ${ }^{1,3,6}$, Benedetta Conte ${ }^{1,3,7}$, Maria Vidal (D) ${ }^{1,2,3}$, Barbara Adamo ${ }^{1,2,3}$, Antoni Martínez (iD) ${ }^{5}$, Montserrat Muñoz ${ }^{1,2,3}$, Reinaldo Moreno ${ }^{1,2,3}$, Patricia Villagrasa ${ }^{1}$, Fernando Salvador ${ }^{1}$, Eva M. Ciruelos ${ }^{1,8}$, Iris Faull $\mathbb{( D D}^{9}$, Justin I. Odegaard ${ }^{9}$ and Aleix Prat $\mathbb{D}^{1,2,3,10 凶}$

Circulating tumor DNA (ctDNA) levels may predict response to anticancer drugs, including CDK4/6 inhibitors and endocrine therapy combinations (CDK4/6i+ET); however, critical questions remain unanswered such as which assay or statistical method to use. Here, we obtained paired plasma samples at baseline and week 4 in 45 consecutive patients with advanced breast cancer treated with CDK4/6i+ET. ctDNA was detected in $96 \%$ of cases using the $74-g e n e$ Guardant 360 assay. A variant allele fraction ratio (VAFR) was calculated for each of the 79 detected mutations between both timepoints. Mean of all VAFRs (mVAFR) was computed for each patient. In our dataset, mVAFR was significantly associated with progression-free survival (PFS). Baseline VAF, on-treatment VAF or absolute changes in VAF were not associated with PFS, nor were CA-15.3 levels at baseline, week 4 or the CA-15.3 ratio. These findings demonstrate that ctDNA dynamics using a standardized multi-gene panel and a unique methodological approach predicts treatment outcome. Clinical trials in patients with an unfavorable ctDNA response are needed.

npj Breast Cancer (2021)7:8; https://doi.org/10.1038/s41523-021-00218-8

In hormone receptor positive (HR+)/HER2-negative advanced breast cancer (BC), CDK4/6i plus ET have remarkably improved survival outcomes and are now considered a standard treatment for most patients ${ }^{1}$. Although this is good news for patients suffering from metastatic BC, improving the efficacy of CDK4/6i and ET using novel treatment strategies might be challenging. On the one hand, no predictive biomarker exists to date to select patients who are going to progress early ${ }^{2}$. On the other hand, improving survival outcomes with new or additional therapies when the control arm has a median PFS of 25-27 months in the first-line setting will require huge personal, physical, and economic resources as well as long periods of follow-up ${ }^{3-5}$. This issue is not restricted to advanced BC but also other cancer types such as lung cancer.

Detection of ctDNA levels before and during therapy might improve CDK4/6i plus ET efficacy, stratify patients, and help design future trials with novel treatment strategies ${ }^{6,7}$. O'Leary and colleagues evaluated early ctDNA dynamics in patients with PIK3CA-mutated HR+/HER2-negative metastatic $B C$ treated with palbociclib and fulvestrant in PALOMA-3 trial ${ }^{8}$. A multiplex digital PCR assay was used and hotspot PIK3CA mutations in exons 9 and 20 were evaluated in plasma ${ }^{8}$. PIK3CA mutation levels from baseline to day 15 of therapy were associated with PFS independently of the treatment received. However, only $22 \%$ of patients with $\mathrm{HR}+/ \mathrm{HER} 2$-negative advanced $\mathrm{BC}$ had detectable PIK3CA mutations in plasma. To circumvent this problem, others argue that individualized gene panels according to each patient's tumor's genetic profile should be prioritized ${ }^{9}$.
We hypothesized that a standardized plasma-based sequencing assay that analyzes multiple genes simultaneously at baseline and after 4 weeks (cycle 2 day 1 [C2D1]) of CDK4/6i plus ET can identify patients with $\mathrm{HR}+/ \mathrm{HER} 2$-negative advanced disease with different treatment outcomes. To accomplish this, we undertook a prospective study from May/2016 to June/2019 of 50 consecutive pre and postmenopausal patients with metastatic HR+/HER2negative $B C$ treated as per standard practice with CDK4/6i and ET (Fig. 1a). Plasma samples were sequenced using the standardized Guardant360 assay v2.11, which can identify single nucleotide variants (SNV) and indels from 74 genes (Fig. 1b) ${ }^{10}$. Among 50 patients, 2 patients (4\%) had insufficient plasma volume, 2 patients (4\%) had missing samples and 1 patient (2\%) was treated in the adjuvant setting after resection of a supraclavicular lymph node and was excluded. Finally, 45 patients (90\%) were evaluable of whom $43(96 \%)$ had ctDNA detectable at some level. Of those 45, 31 (69\%) had ctDNA-positive disease (i.e. highest VAF detected $\geq \operatorname{VAF} 0.4 \%$ at some timepoint) and $14(31 \%)$ were considered to have ctDNA-low disease (i.e. highest VAF detected $<0.4 \%$ or nondetected at both timepoints). Of the 31 ctDNA-positive patients, 30 had ctDNA-positive disease at baseline and 1 had ctDNA-low at baseline but ctDNA-positive at cycle 2 (Fig. 1c and Table 1). Mutations in 42 genes were identified at baseline and the 4 most frequent altered genes were PIK3CA, ESR1, TP53, and ATM (Fig. 1d); $\geq 1$ mutation with $\geq \operatorname{VAF} 0.4 \%$ in any of these 4 genes was found in 24 patients $(53.3 \%)$. We had available tissue samples collected from archival biopsies before treatment with CDK4/6i plus ET in 26 patients. As shown in previous studies, all intrinsic molecular

\footnotetext{
${ }^{1}$ SOLTI Cancer Research Group, Barcelona, Spain. ${ }^{2}$ Department of Medical Oncology, Hospital Clinic of Barcelona, Barcelona, Spain. ${ }^{3}$ Translational Genomics and Targeted Therapies in Solid Tumors, August Pi i Sunyer Biomedical Research Institute (IDIBAPS), Barcelona, Spain. ${ }^{4}$ Lineberger Comprehensive Cancer Center, University of North Carolina at Chapel Hill, Chapel Hill, NC, USA. ${ }^{5}$ Department of Pathology, Hospital Clinic of Barcelona, Barcelona, Spain. ${ }^{6}$ Department of Clinical Medicine and Surgery, University of Naples Federico II, Naples, Italy. ${ }^{7}$ Department of Medical Oncology U.O. Oncologia Medica 2, IRCCS Ospedale Policlinico San Martino, Genova, Italy. ${ }^{8}$ Department of Medical Oncology, Hospital Universitario 12 de Octubre, Madrid, Spain. ${ }^{9}$ Guardant Health, Inc., Redwood City, CA, USA. ${ }^{10}$ Department of Medicine, University of Barcelona, Barcelona, Spain. 凶email: alprat@clinic.cat
} 
a

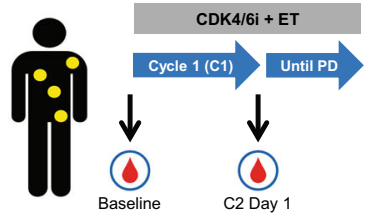

C

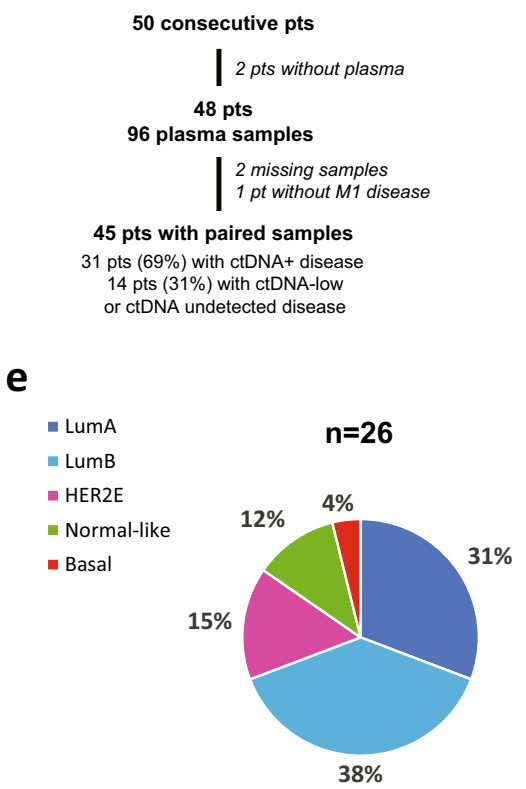

b

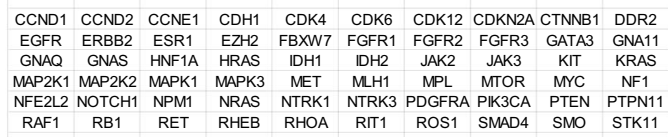

d

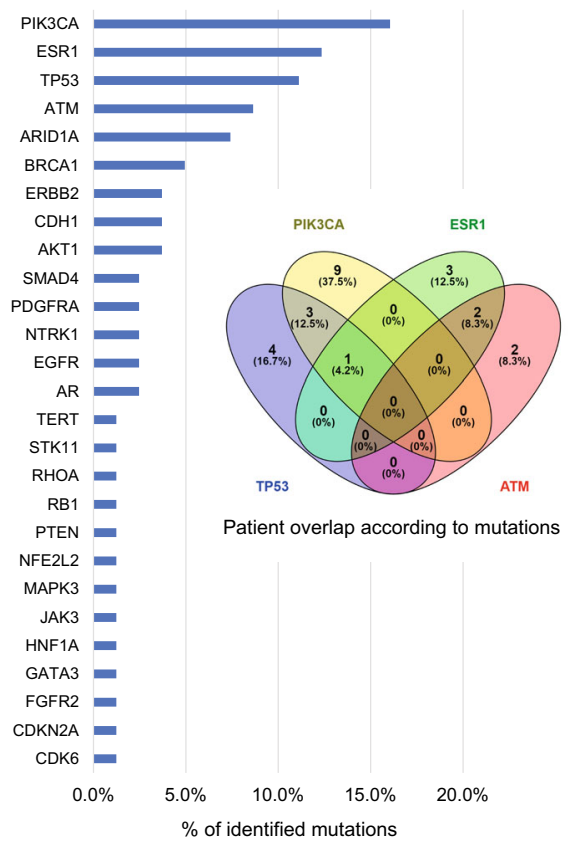

Fig. 1 Description of the study. a Blood samples were extracted at baseline and after 1 cycle (i.e. 4 weeks) in patients with metastatic $\mathrm{HR}+/$ HER2-negative BC treated with CDK4/6i plus ET. b The list of 74 genes analyzed by Guardant360. c CONSORT diagram. d Frequency of gene mutations with $\geq \operatorname{VAF} 0.4 \%$ at baseline identified in the patient dataset and Venn diagram with the 4 most frequent mutations. e PAM50 distribution (LumA = Luminal A, LumB=Luminal B, HER2E = HER2-enriched). *PD Progressive disease, pts patients, M1 metastatic.

subtypes were identified using the PAM50 subtype predictor, although Luminal A and B subtypes predominated (Fig. 1e) ${ }^{11,12}$.

A total of 159 mutations (SNV and indels) were found at baseline; of them, 93 were detected in C2D1. From the 159 mutations in baseline, 79 had a VAF $\geq 0.4 \%$ in 31 patients (mean of 2.6 alterations per patient), 60 of these mutations were detected at some level in C2D1. 31 mutations were detected in C2D1 and not in baseline, but only 3 mutations with $\mathrm{VAF} \geq 0.4 \%$ were detected at C2D1 and not in baseline (Supplementary Fig. 3 and Fig. 4). Mean VAF (mVAF) of the 79 mutations was 6.2 at baseline and 5.1 at C2D1 ( $p$-value=0.040) (Fig. 2a). Any decrease in VAF at C2D1 compared to baseline was observed in $71 \%(56 / 79)$ of the tracked mutations. To capture the magnitude of ctDNA response, a VAFR from C2D1 to baseline was calculated for each genetic mutation and a mVAFR was computed for each patient (Fig. 2b and Methods). $35 \%$ of patients had mVAFR of $\leq 0.3$ (mVAFR-low), $29 \%$ had mVAFR of $0.31-0.99$ (mVAFR-medium) and 35\% had a mVAFR of $\geq 1.0$ (mVAFR-high) (Fig. 2c). Finally, no clinical features were found specific of a particular ctDNA group (Table 1).

With a median follow-up of 20.4 months, a significant association between the various ctDNA groups and PFS was observed across all patients ( $p$-value $=0.02$ ) (Fig. $2 \mathrm{~d}$, e). Compared to the mVAFR-high group, the mVAFR-low group was associated with better PFS (not reached (NR) (95\% confidence interval [Cl] 5.40-NR) vs. 4.2 months ( $95 \% \mathrm{Cl} 2.41-11.60)$; adjusted hazard ratio $[\mathrm{aHR}]=0.31,95 \% \mathrm{Cl} 0.09-1, p$-value $=0.049$ ). Similarly, the mVAFRlow and ctDNA-low groups combined was associated with better PFS compared to the mVAFR-high group (33.7 (95\% Cl 5.3-33.7) vs. 4.2 months; $\mathrm{aHR}=0.25,95 \% \mathrm{Cl} 0.09-0.7, p$-value $=0.008)$. The results in ctDNA-low group are in line with what was previously described in other metastatic tumors, as low ctDNA levels seem to be a good prognostic feature. In addition, mVAFR as a continuous variable was also found significantly associated with PFS (aHR per 1 -unit increase $=2.07,95 \% \mathrm{Cl} 1.2-3.5, p$-value $=0.008$ ) (Supplementary Table 2). Of note, mVAF at baseline, or mVAF at C2D1 or absolute changes in mVAF (delta-VAF) were not found associated with PFS when evaluated as continuous variables (Fig. $2 f$ and Supplementary Table 1). These results are in consonance with different studies that assessed ctDNA dynamics and have showed that higher pretreatment VAF acts as poorer prognostic factor but has no predictive value ${ }^{13,14}$. On the other hand, there is not a standard method yet to assess ctDNA dynamics; several studies have used the delta-VAF or a ratio between the two timepoints $8,13,15-17$. However, while delta-VAF is able to stratify patients similarly to on-treatment $\mathrm{VAF}$, it only assesses absolute VAF changes and equates patients with low VAF who had a significant decrease in ctDNA level and patients with a higher VAF and smaller decrease in ctDNA level. Interestingly, no patient with non-luminal tumors was identified as high ctDNA responder (i.e. mVAFR-low), consistent with previous reports in advanced HR +/HER2-negative disease associating the Luminal phenotype with better prognosis and response to ET compared to non-luminal tumors (Fig. 2g) ${ }^{18,19}$.

At baseline, median CA-15.3 value was $45 \mathrm{U} / \mathrm{mL}(7-6,672)$, and 26 patients $(61 \%)$ had high CA-15.3 values $(>35 \mathrm{U} / \mathrm{mL})$. At C2D1, median CA-15.3 value was $42 \mathrm{U} / \mathrm{mL}$ (range $8-9,868$ ), and 19 
Table 1. Clinical features of the patient dataset according to ctDNA levels and dynamics.

\begin{tabular}{|c|c|c|c|c|}
\hline & $\begin{array}{l}\text { All patients } \\
N=45\end{array}$ & $\begin{array}{l}\text { ctDNA-low } \\
N=14\end{array}$ & $\begin{array}{l}\text { mVAFR-low } \\
N=11\end{array}$ & $\begin{array}{l}\text { mVAFR- } \\
\text { med/high } \\
N=20\end{array}$ \\
\hline \multicolumn{5}{|l|}{ Age } \\
\hline $\begin{array}{l}\text { Median } \\
\text { (range) yr }\end{array}$ & $61.4(39-87)$ & $59.7(39-87)$ & $56.9(45-72)$ & $64(42-75)$ \\
\hline $\begin{array}{l}<65 \mathrm{yr}- \\
\text { no. }(\%)\end{array}$ & $28(62 \%)$ & $8(57 \%)$ & $9(82 \%)$ & $11(55 \%)$ \\
\hline $\begin{array}{l}\geq 65 \mathrm{yr}- \\
\text { no. (\%) }\end{array}$ & $17(38 \%)$ & $6(43 \%)$ & $2(18 \%)$ & $9(45 \%)$ \\
\hline \multicolumn{5}{|l|}{ Line-no. (\%) } \\
\hline First & $21(47 \%)$ & $8(57 \%)$ & $5(45 \%)$ & $9(45 \%)$ \\
\hline Second & $16(36 \%)$ & $5(36 \%)$ & $5(45 \%)$ & $5(25 \%)$ \\
\hline $\begin{array}{l}\text { Third } \\
\text { or more }\end{array}$ & $8(18 \%)$ & $1(7 \%)$ & $1(9 \%)$ & $6(30 \%)$ \\
\hline \multicolumn{5}{|c|}{ ECOG-PS ${ }^{a}-$ no. (\%) } \\
\hline 0 & 17 (38\%) & $5(36 \%)$ & $5(45 \%)$ & 7 (35\%) \\
\hline 1 & $27(60 \%)$ & $9(64 \%)$ & $6(55 \%)$ & $12(60 \%)$ \\
\hline 2 & $1(2 \%)$ & 0 & 0 & $1(5 \%)$ \\
\hline \multicolumn{5}{|c|}{ Endocrine therapy-no. (\%) } \\
\hline $\begin{array}{l}\text { Aromatase } \\
\text { inhibitor }\end{array}$ & 15 (33\%) & $4(29 \%)$ & $3(27 \%)$ & $8(40 \%)$ \\
\hline Fulvestrant & $26(58 \%)$ & $9(64 \%)$ & 7 (64\%) & $10(50 \%)$ \\
\hline Tamoxifen & $4(9 \%)$ & $1(7 \%)$ & $1(9 \%)$ & $1(5 \%)$ \\
\hline \multicolumn{5}{|c|}{ Type of CDK4/6 inhibitor-no. (\%) } \\
\hline Palbociclib & $40(89 \%)$ & $12(86 \%)$ & 10 (91\%) & $18(90 \%)$ \\
\hline Ribociclib & $5(11 \%)$ & $2(14 \%)$ & $1(9 \%)$ & $2(10 \%)$ \\
\hline \multicolumn{5}{|c|}{ Disease site-no. (\%) } \\
\hline Visceral & $2(64 \%)$ & $10(71 \%)$ & 7 (64\%) & $12(60 \%)$ \\
\hline Non visceral & $16(36 \%)$ & $4(29 \%)$ & $4(36 \%)$ & $8(40 \%)$ \\
\hline Bone-only & $10(22 \%)$ & $1(7 \%)$ & $4(36 \%)$ & $5(25 \%)$ \\
\hline \multicolumn{5}{|c|}{ Number of metastatic locations-no. (\%) } \\
\hline$<3$ & $23(51 \%)$ & $8(57 \%)$ & 7 (64\%) & $8(40 \%)$ \\
\hline$\geq 3$ & $22(49 \%)$ & $6(43 \%)$ & $4(36 \%)$ & $12(60 \%)$ \\
\hline \multicolumn{5}{|c|}{ "De novo" metastasis-no. (\%) } \\
\hline No & $33(73 \%)$ & $10(71 \%)$ & 7 (64\%) & $15(75 \%)$ \\
\hline Yes & $12(27 \%)$ & $4(29 \%)$ & $4(36 \%)$ & $5(25 \%)$ \\
\hline \multicolumn{5}{|c|}{ Prior hormone sensitivity—no. (\%) } \\
\hline Sensitivity & $31(69 \%)$ & $9(64 \%)$ & $7(64 \%)$ & $15(75 \%)$ \\
\hline Resistance $^{\mathrm{b}}$ & $14(31 \%)$ & $5(36 \%)$ & $4(36 \%)$ & $5(25 \%)$ \\
\hline \multicolumn{5}{|c|}{ Histology—no. (\%) } \\
\hline Ductal & $32(71 \%)$ & $11(79 \%)$ & $8(73 \%)$ & $13(65 \%)$ \\
\hline Lobular & $9(20 \%)$ & $3(21 \%)$ & $3(27 \%)$ & $3(15 \%)$ \\
\hline Other & $4(9 \%)$ & 0 & 0 & $4(20 \%)$ \\
\hline \multicolumn{5}{|l|}{ Grade-no. (\%) } \\
\hline 1 & $5(11 \%)$ & $2(14 \%)$ & $2(18 \%)$ & $1(5 \%)$ \\
\hline 2 & $19(42 \%)$ & $5(36 \%)$ & $6(55 \%)$ & $8(40 \%)$ \\
\hline 3 & $12(27 \%)$ & $5(36 \%)$ & $2(18 \%)$ & $5(25 \%)$ \\
\hline Unknown & $9(20 \%)$ & $2(14 \%)$ & $1(9 \%)$ & $6(30 \%)$ \\
\hline \multicolumn{5}{|c|}{ Ki67 (\%)—no. (\%) } \\
\hline $1-14$ & $9(20 \%)$ & $4(29 \%)$ & $2(18 \%)$ & $3(15 \%)$ \\
\hline $15-20$ & $3(7 \%)$ & $1(7 \%)$ & 0 & $2(10 \%)$ \\
\hline$>20$ & $25(56 \%)$ & $7(50 \%)$ & $6(55 \%)$ & $12(60 \%)$ \\
\hline Unknown & $8(18 \%)$ & $2(14 \%)$ & $3(27 \%)$ & $3(15 \%)$ \\
\hline
\end{tabular}

apS: performance status.

${ }^{\mathrm{b}}$ Hormone resistance defined as relapse while on the first 2 years of adjuvant $\mathrm{ET}$, or progression of disease within first 6 months of first-line $\mathrm{ET}$ for advanced BC, while on ET. patients (51\%) had high CA-15.3 values. No significant differences in CA-15.3 levels were observed between baseline and C2D1 ( $p$ value $=0.350$ ). The median ratio of CA-15.3 between C2D1 and baseline was 1.05 (range 0.4-1.6). No correlation was observed between CA-15.3 ratio and ctDNA mVAFR (correlation coefficient $=-0.021)$. The levels of CA-15.3 at baseline or C2D1, and the CA15.3 ratio, were not found associated with PFS (data not shown).

Our study has limitations worth noting. First, the limited sample size, which precludes more in-depth analysis within subgroups of patients. For example, identification of an optimal mVAFR cutoff to define prognosis will require a larger sample set. Second, there is not yet a standardized method to assess the ctDNA dynamics. We are aware that the arithmetic mean of VAFRs could result in overestimation of the average ctDNA change due to the nonadditive nature of the ratios and the small number of detected mutations per patient. To address this, we assigned fixed VAFR values of 10 or 0.1 (Methods). As this mitigation strategy could not cover all potential cases, we have studied other possibility taking the logarithm of each ratio, and then taking the mean of logarithms. The correlation coefficient between both scores (i.e. simple arithmetic mean ratio and logarithm mean ratio) was 0.94 and the Kappa concordance score between mVAFR-based groups of patients and mVAFR_log-based groups of patients, 0.88 . As expected, this scoring method was also found associated with PFS (Supplementary Section). The best method to assess ctDNA dynamics should be determined in future studies. Third, the short follow-up time does not allow associations with overall survival. Fourth, we did not explore if tracking ctDNA levels of particular genes is better than tracking any detected altered gene in plasma. Nonetheless, a strong argument in favor of our approach is that it does not rely on specific genes but rather on the dynamic changes of the altered genes identified before initiating treatment. Fifth, it is unclear if this approach and methods will be applicable for other therapies or other cancer types. Lastly, we cannot completely exclude that some of the alterations identified are from clonal hematopoyesis $(\mathrm{CH})$. However, we try to correct for clinical $\mathrm{CH}$ in two ways. First, we excluded any variant present at $<0.4 \%$ since the vast majority of $\mathrm{CH}$ variants are present below this level and this excludes them from contributing to the assessment. Second, our method averages all variants present, which dilutes the impact of any atypical $\mathrm{CH}$ variant that may have exceeded the VAF threshold. In such cases, the $\mathrm{CH}$ variant would remain unaffected by therapy and remain at a static VAF and, as such, would not contribute to any changes in tumor ctDNA fraction, although it could dilute somewhat any changes observed in true tumor ctDNA. Finally, our findings will require further validation in patients with advanced BC treated with CDK4/6i plus ET.

Our findings have several potential clinical implications. Most importantly, they suggest that early ctDNA dynamics using a multi-gene assay and a particular statistical methodology serve as a general biomarker to identify patients with advanced BC who are at high risk of progression during standard therapy with CDK4/ $6 \mathrm{i}$ and ET, giving the opportunity to intervene and change the treatment or add another treatment early. Notably, the biomarker seems independent of baseline clinical features, tumor marker CA15.3 and the clinical setting, and the relationship of low ctDNAresponders with the non-luminal subtypes is weak. It will be important to also assess its value on the outcome of additional therapies and cancer types. Overall, our findings support the notion that monitoring ctDNA should be an integral part during drug development and should allow the design of novel clinical trials in key patient populations, such as those patients with an unfavorable ctDNA response. 
a

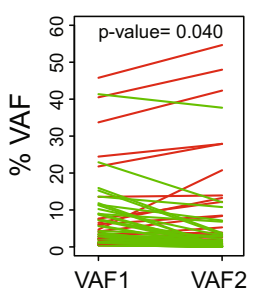

d

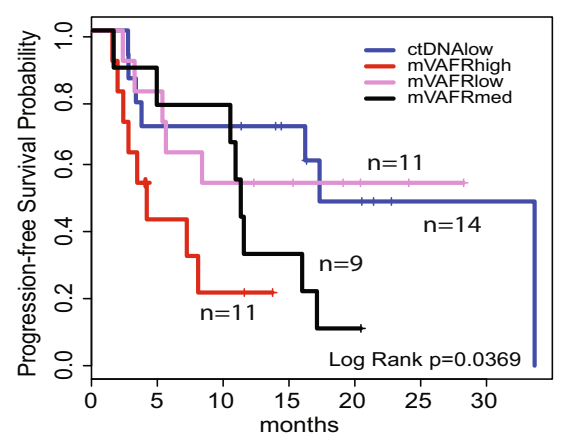

f

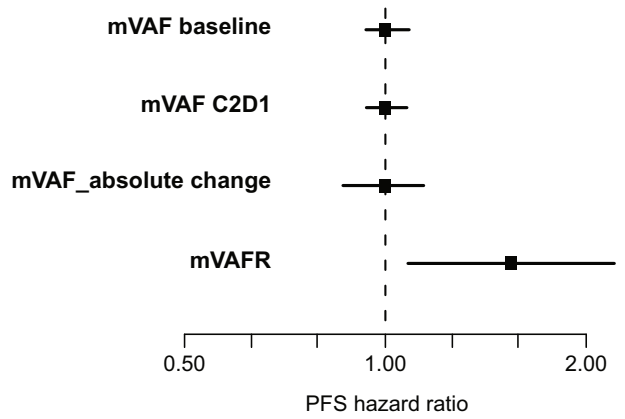

b

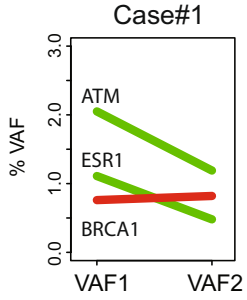

ATM VAF ratio $=0.58$ ESR1 VAF ratio $=0.43$ BRCA VAF ratio $=1.08$

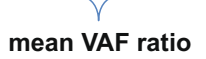

$(\mathrm{mVAFR})=\mathbf{0 . 7 0}$
C

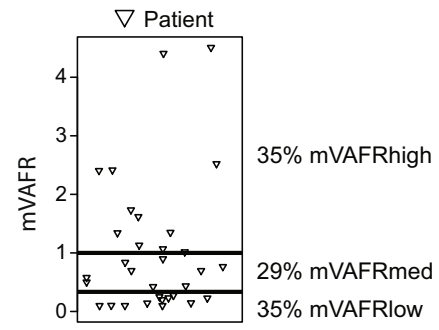

e

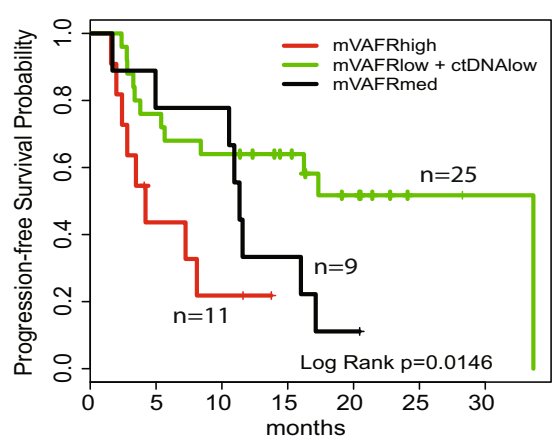

g

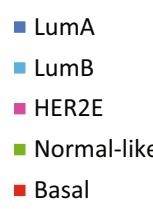

- Basal

mVAFRmed/high $\mathrm{n}=11$

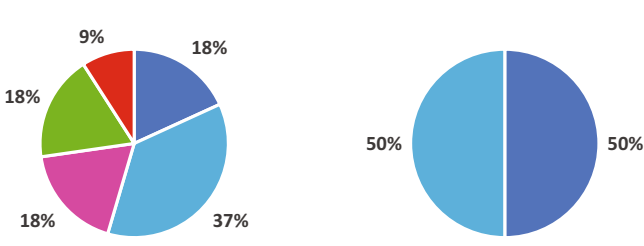

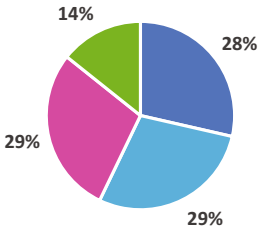

ctDNA-low $n=7$

mVAFRlow $n=8$

Fig. 2 ctDNA dynamics and survival outcome. a VAF changes of 79 detected mutations from baseline to C2D1. $p$-value was calculated with Wilcoxon rank sum test. b lllustration of a case and its mVAFR. c Distribution plot of patients with mVAFR-low (mVAFR of $\leq 0.3)$, mVAFR-medium [mVAFRmed] (mVAFR of 0.31-0.99) and mVAFR-high (mVAFR of $\geq 1.0$ ). d PFS based on ctDNA dynamics. e PFS based on ctDNA dynamics after combining the VAFR-low and ctDNA-low groups. $f$ PFS hazard ratio forest plots across 4 different methods of assessing mVAF as a continuous variable: mVAF baseline, $\mathrm{mVAF}$ at C2D1, absolute change of mVAF between baseline and C2D1 and mVAFR of all mutations with a VAF $\geq 0.4$ at baseline or C2D1. g Distribution of the intrinsic subtypes in the mVAFR-low group ( $n=4$ Luminal A [LumA], $n=4$ Luminal B [LumB], $n=3$ nonavailable tissue [NA]), mVAFR-medium/high group $(n=2$ LumA, $n=4$ LumB, $n=2$ HER2-Enriched [HER2E], $n=2$ Normal-like, $n=1$ Basal-like, $n=9 \mathrm{NA}$ ) and ctDNA-low group ( $n=2$ LumA, $n=2 \mathrm{LumB}, n=2$ HER2E, $n=2$ Normal-like, $n=7 \mathrm{NA}$ ).

\section{METHODS}

\section{Study design and patients}

This is a prospective, single-center study in 50 consecutive patients with advanced $B C$. Eligible patients were $\geq 18$ years of age with histologically confirmed HR+/HER2-negative inoperable or metastatic BC treated with a CDK4/6i and ET. Blood samples for sample collection were obtained at baseline and at C2D1. Clinical data, results of computed tomography (CT) imaging, and serial blood samples were collected as per standard practice. The study was performed in accordance with Good Clinical Practice guidelines and the World Medical Association Declaration of Helsinki. The study was approved by the local institutional research ethics committee, and all patients provided written informed consent.

\section{Plasma samples}

Approximately $30 \mathrm{~mL}$ of venous blood was extracted at each timepoint and collected in EDTA tubes. Blood was processed within $2 \mathrm{~h}$ after the collection. Centrifugation at $1600 \mathrm{~g}$ for 10 minutes at $4{ }^{\circ} \mathrm{C}$ was performed to separate the plasma from the peripheral-blood cells. We obtained approximately $12 \mathrm{~mL}$ of plasma per patient and timepoint, and plasma was immediately aliquoted in $1.5 \mathrm{~mL}$ tubes and then we centrifugated them at $16,000 \mathrm{~g}$ at $4{ }^{\circ} \mathrm{C}$ for another 10 minutes to remove the residual supernatant and any remaining contaminants including cells. Separated plasma was aliquoted in a $1.5 \mathrm{~mL}$ tube and immediately stored in a deep freezer at $-80^{\circ} \mathrm{C}$.

Cell-free DNA (cfDNA) was extracted from $1.5 \mathrm{ml}$ aliquots of plasma using the QIAamp circulating nucleic acid kit (Qiagen), concentrated using 
Agencourt Ampure XP beads (Beckman Coulter), and quantified by Qubit fluorometer (Life Technologies, Carlsbad, CA, USA). All cfDNA isolation and sequencing was performed at Guardant Health (Redwood City, CA, USA).

\section{DNA sequencing}

Genomic alterations (SNV, insertions and deletions (indels) and amplifications) were detected from cfDNA extracted from plasma samples using a broad targeted NGS-based 74-gene panel (Guardant360), including coverage of the most prevalent tumor suppressor genes in human cancers (Fig. 1b). After isolation of cfDNA by hybrid capture, the assay was performed using molecular barcoding and proprietary bioinformatics algorithms with massively parallel sequencing on an Illumina Hi-Seq 2500 platform in a CLIA/CAP accredited laboratory (Guardant Health; Redwood City, CA, USA).

\section{ctDNA response definition}

We filtered somatic mutations with $\mathrm{VAF} \geq 0.4 \%$ either at baseline (C1D1) or C2D1, based on $95 \%-100 \%$ limits of detection for this technology. Those patients with $\mathrm{VAF}<0.4 \%$ at both timepoints were considered low-shedding tumors. We calculated the proportional change for all variants detected between the 2 timepoints (VAF ratio [VAFR] =VAF C2D1/VAF C1D1). For variants detected at 1 timepoint but not the other, VAF was set to 0 . We considered all undetected variants at $\mathrm{C} 2 \mathrm{D} 1$, or variants with a $\mathrm{VAF}<0.4 \%$ at C2D1, to have a VAFR of 0.1 as minimum. The reason is to prevent skewing of the average by variance introduced by quantitation variability below $0.4 \%$ VAF. We considered all new variants detected at C2D1 but not at baseline to have a VAFR of 10 as maximum. The reason is to prevent skewing of the average by variance introduced by quantitation variability below $0.4 \%$ and by dividing by numbers that approach zero. Finally, a mVAFR was calculated per patient taking the average of all VAFR.

\section{CA-15.3 determination}

The CA-15.3 assay was performed by the BRAHMS Kryptor Plus compact controller using TRACE (Time-Resolved Amplified Cryptate Emission) technology. CA-15.3 was considered elevated when it was above the normal upper limit $(35 \mathrm{U} / \mathrm{mL})$.

\section{PAM50 subtype determination}

A minimum of $\sim 125 \mathrm{ng}$ of total RNA from formalin-fixed paraffin embedded tumor samples was used to measure the expression of the 50 PAM50 subtype predictor genes and 5 housekeeping genes using the nCounter platform (Nanostring Technologies, Seattle, USA).

\section{Response evaluation by image}

$\mathrm{CT}$ scan and bone scintigraphy were performed as per standard practice. RECIST 1.1 was used to evaluate tumor responses.

\section{Statistical analysis}

The primary objective was to evaluate the association of ctDNA dynamics from baseline to C2D1 and PFS. PFS was defined as the time from initiation of treatment until progression or death. Univariate and multivariate Cox proportional hazard regression analysis was used to investigate the association of each variable with PFS. VAF changes between timepoints were calculated with Wilcoxon Rank Sum Test. The significance level was set to a 2-sided alpha of 0.05. $p$-value was calculated with Wilcoxon Rank Sum Test. All analyses were performed with R code 3.6.

\section{Reporting summary}

Further information on research design is available in the Nature Research Reporting Summary linked to this article.

\section{DATA AVAILABILITY}

The data generated and analyzed during this study are described in the following data record: https://doi.org/10.6084/m9.figshare.13365521 ${ }^{20}$. The ctDNA and clinical data are available in two separate tabs in the Excel spreadsheet "ctDNA and clinical dataset.xlsx", which is openly available and shared as part of the figshare data record $^{20}$. The 'CDK series - ctDNA and clinicopathological dataset' is not publicly available in order to protect patient privacy. Requests for access to this dataset can be made to the corresponding author.
Received: 29 July 2020; Accepted: 7 January 2021; Published online: 03 February 2021

\section{REFERENCES}

1. Schettini, F. et al. Overall survival of CDK4/6-inhibitors-based treatments in clinically relevant subgroups of metastatic breast cancer: systematic review and meta-analysis. J. Natl. Cancer Inst. 112, 1089-1097 (2020).

2. Schoninger, S. F. \& Blain, S. W. The ongoing search for biomarkers of CDK4/6 inhibitor responsiveness in breast cancer. Mol. Cancer Ther. 19, 3-12 (2020).

3. Hortobagyi, G. N. et al. Updated results from MONALEESA-2, a phase III trial of firstline ribociclib plus letrozole versus placebo plus letrozole in hormone receptorpositive, HER2-negative advanced breast cancer. Ann. Oncol. 29, 1541-1547 (2018).

4. Rugo, H. S. et al. Palbociclib plus letrozole as first-line therapy in estrogen receptor-positive/human epidermal growth factor receptor 2-negative advanced breast cancer with extended follow-up. Breast Cancer Res. Treat. https://doi.org/ 10.1007/s10549-018-05125-4 (2019).

5. Johnston, S. et al. MONARCH 3 final PFS: a randomized study of abemaciclib as initial therapy for advanced breast cancer. npj Breast Cancer https://doi.org/ 10.1038/s41523-018-0097-z (2019).

6. Heitzer, E., Ulz, P. \& Geigl, J. B. Circulating tumor DNA as a liquid biopsy for cancer. Clin. Chem. https://doi.org/10.1373/clinchem.2014.222679 (2015).

7. Wan, J. C. M. et al. Liquid biopsies come of age: Towards implementation of circulating tumour DNA. Nat. Rev. Cancer https://doi.org/10.1038/nrc.2017.7 (2017)

8. O'Leary, B. et al. Early circulating tumor DNA dynamics and clonal selection with palbociclib and fulvestrant for breast cancer. Nat. Commun. 9, 1-10 (2018).

9. Vandeputte, C. et al. Circulating tumor DNA in early response assessment and monitoring of advanced colorectal cancer treated with a multikinase inhibitor. Oncotarget https://doi.org/10.18632/oncotarget.24879 (2018).

10. Lanman, R. B. et al. Analytical and clinical validation of a digital sequencing panel for quantitative, highly accurate evaluation of cell-free circulating tumor DNA. PLOS ONE 10, 1-27 (2015).

11. Cejalvo, J. M. et al. Clinical implications of the non-luminal intrinsic subtypes in hormone receptor-positive breast cancer. Cancer Treat. Rev. https://doi.org/ 10.1016/j.ctrv.2018.04.015 (2018).

12. Prat, A. et al. Ribociclib plus letrozole versus chemotherapy for postmenopausal women with hormone receptor-positive, HER2-negative, luminal B breast cancer (CORALLEEN): an open-label, multicentre, randomised, phase 2 trial. Lancet Oncol. https://doi.org/10.1016/S1470-2045(19)30786-7 (2020).

13. Zhang, Q. et al. Prognostic and predictive impact of circulating tumor DNA in patients with advanced cancers treated with immune checkpoint blockade. Cancer Discov. CD-20-0047 https://doi.org/10.1158/2159-8290.cd-20-0047 (2020).

14. Goodall, J. et al. Circulating tumor DNA (ctDNA) dynamics associate with treatment response and radiological progression-free survival (rPFS): analyses from a randomized phase II trial in metastatic castration-resistant prostate cancer (mCRPC). J. Clin. Oncol. https://doi.org/10.1200/jco.2020.38.15_suppl.5508 (2020).

15. Raja, R. et al. Early reduction in ctDNA predicts survival in patients with lung and bladder cancer treated with durvalumab. Clin. Cancer Res. 24, 6212-6222 (2018).

16. Parkinson, C. A. et al. Exploratory analysis of TP 53 mutations in circulating tumour DNA as biomarkers of treatment response for patients with relapsed high-grade serous ovarian carcinoma: a retrospective study. PLoS Med. 13, 1-25 (2016).

17. Garlan, F. et al. Early evaluation of circulating tumor DNA as marker of therapeutic efficacy in metastatic colorectal cancer patients (PLACOL study). Clin. Cancer Res. 23, 5416-5425 (2017).

18. Finn, R. S. et al. Biomarker analyses of response to cyclin-dependent kinase $4 / 6$ inhibition and endocrine therapy in women with treatment-naïve metastatic breast cancer. Clin. Cancer Res. 26, 110-121 (2020).

19. $M a, C$. X. et al. NeoPalAna: Neoadjuvant palbociclib, a cyclin-dependent kinase $4 / 6$ inhibitor, and anastrozole for clinical stage 2 or 3 estrogen receptor-positive breast cancer. Clin. Cancer Res. https://doi.org/10.1158/1078-0432.CCR-16-3206 (2017).

20. Martínez-Sáez, O. et al. Metadata record for the manuscript: Circulating tumor DNA dynamics using a standardized multi-gene panel in advanced breast cancer treated with CDK4/6 inhibition and endocrine therapy. figshare https://doi.org/ 10.6084/m9.figshare.13365521 (2020).

\section{ACKNOWLEDGEMENTS}

This study was funded by Instituto de Salud Carlos III (PI19/01846) (to A.P.), Instituto de Salud Carlos III (PI18/01408) (to E.C.), Breast Cancer Research Foundation (to A.P.), PhD4MD (to N.C.), Fundació La Marató TV3 (to A.P), RESCUER Horizon 2020 (to A.P.), Save the Mama (to A.P.), Pas a Pas (to A.P.), Asociación Cáncer de Mama Metastásico (to A.P.), Fundación Científica Asociación Española Contra el Cáncer (to F.B.M.) and Fundación SEOM (SEOM 2018 Grant: Fellowship for Training in Research in Reference 
Centers) (to T.P.). The study was designed by investigators from Hospital Clinic. Funding sources had no role in the design and conduction of this study, and in the analysis and interpretation of data. Guardant Health provided the assay. All authors had full access to all data in the study and had final responsibility for the decision to submit for publication.

\section{AUTHOR CONTRIBUTIONS}

All authors made substantial contributions to the conception or design of the work or the acquisition, analysis, or interpretation of the data. All authors drafted the work or revised it critically for important intellectual content and gave final approval of the completed version. All authors have accountability for all aspects of the work in ensuring that questions related to the accuracy or integrity of any part of the work are appropriately investigated and resolved.

\section{COMPETING INTERESTS}

The authors declare no competing interests.

\section{ADDITIONAL INFORMATION}

Supplementary information The online version contains supplementary material available at https://doi.org/10.1038/s41523-021-00218-8.
Correspondence and requests for materials should be addressed to A.P.

Reprints and permission information is available at http://www.nature.com/ reprints

Publisher's note Springer Nature remains neutral with regard to jurisdictional claims in published maps and institutional affiliations.

(i) Open Access This article is licensed under a Creative Commons Attribution 4.0 International License, which permits use, sharing, adaptation, distribution and reproduction in any medium or format, as long as you give appropriate credit to the original author(s) and the source, provide a link to the Creative Commons license, and indicate if changes were made. The images or other third party material in this article are included in the article's Creative Commons license, unless indicated otherwise in a credit line to the material. If material is not included in the article's Creative Commons license and your intended use is not permitted by statutory regulation or exceeds the permitted use, you will need to obtain permission directly from the copyright holder. To view a copy of this license, visit http://creativecommons. org/licenses/by/4.0/.

(c) The Author(s) 2021 